\title{
High-Energy Collision of Two Black Holes
}

\author{
Ulrich Sperhake, ${ }^{1}$ Vitor Cardoso, ${ }^{2,3}$ Frans Pretorius, ${ }^{4}$ Emanuele Berti, ${ }^{5}$ and José A. González ${ }^{6}$ \\ ${ }^{1}$ Theoretisch Physikalisches Institut, Friedrich Schiller Universität, 07743 Jena, Germany \\ ${ }^{2}$ CENTRA, Departamento de Física, Instituto Superior Técnico, Av. Rovisco Pais 1, 1049-001 Lisboa, Portugal \\ ${ }^{3}$ Department of Physics and Astronomy, The University of Mississippi, University, Mississippi 38677-1848, USA \\ ${ }^{4}$ Department of Physics, Princeton University, Princeton, New Jersey 08544, USA \\ ${ }^{5}$ Jet Propulsion Laboratory, California Institute of Technology, Pasadena, California 91109, USA \\ ${ }^{6}$ Instituto de Física y Matemáticas, Universidad Michoacana de San Nicolás de Hidalgo, \\ Edificio C-3, Cd. Universitaria. C. P. 58040 Morelia, Michoacán, Mexico \\ (Received 10 June 2008; published 14 October 2008)
}

\begin{abstract}
We study the head-on collision of two highly boosted equal mass, nonrotating black holes. We determine the waveforms, radiated energies, and mode excitation in the center of mass frame for a variety of boosts. For the first time we are able to compare analytic calculations, black-hole perturbation theory, and strong field, nonlinear numerical calculations for this problem. Extrapolation of our results, which include velocities of up to $0.94 c$, indicate that in the ultrarelativistic regime about $14 \pm 3 \%$ of the energy is converted into gravitational waves. This gives rise to a luminosity of order $10^{-2} c^{5} / G$, the largest known so far in a black-hole merger.
\end{abstract}

DOI: 10.1103/PhysRevLett.101.161101

PACS numbers: 04.25.dc, 04.25.dg, 04.70.-s

Introduction.-An important and long-standing problem in general relativity concerns the ultrarelativistic scattering of black holes (BHs). This is one of the most violent events one can conceive of in the theory. The lack of solutions has spurred much speculation about what may happen in this regime. For example, these events are a natural testing ground for the cosmic censorship conjecture: is there a class of initial conditions where they generically lead to the formation of a naked singularity, or do event horizons always form to cloth singular behavior in the geometry?

Related questions concern the ultrarelativistic scattering of particles. If the center of mass (c.m.) energy is beyond the Planck scale, gravity is expected to dominate the interaction. Furthermore, since the kinetic energy dominates over the rest-mass energy, the gravitational interaction should be rather insensitive to the structure of the particles, implying that the trans-Planckian scattering of point particles should be well described by BH scattering [1]. This is of particular relevance for recent proposals to solve the hierarchy problem by adding "large" extra dimensions [2], or an extra dimension with a warp factor [3], thus producing an effective electroweak Planck scale. This offers the exciting possibility that BHs could be produced in particle colliders and ultra high-energy cosmic ray interactions with the atmosphere $[1,4]$. A naive estimate of the cross section for $M_{\mathrm{Pl}} \sim 1 \mathrm{TeV}$ predicts that super-TeV particle colliders will produce $\mathrm{BHs}$ at a rate of a few per second, making the Large Hadron Collider (LHC) at CERN a potential black-hole factory. An important element to search for $\mathrm{BH}$ production signatures is to understand the $\mathrm{BH}$ scattering process, and, in particular, the energy lost to gravitational radiation. Given that the beam commissioning to $7 \mathrm{TeV}$ is scheduled for late 2008 , this is a timely research topic. Further interesting applica- tions of high-speed $\mathrm{BH}$ collisions to high-energy physics have recently been suggested by the AdS/CFT correspondence conjecture [5]. Particularly intriguing is the possibility of using this duality to understand properties of the quark-gluon plasma formed in gold ion collisions at Brookhaven's Relativistic Heavy Ion Collider (RHIC) through a study of ultrarelativistic BH collisions in AdS [6].

Early attempts to understand the ultrarelativistic $\mathrm{BH}$ scattering problem were based on work by Penrose [7] in the 1970s. He modeled the spacetime metric as the union of two Aichelburg-Sexl waves [8], describing the collision of two infinitely boosted Schwarzschild BHs, and found a closed trapped surface at the moment of collision, giving an upper limit of roughly $29 \%$ of the initial energy of the spacetime radiated in gravitational waves. Beyond the collision event the solution is unknown. Given the extreme conditions of high-speed scattering it is unlikely that analytic solutions describing the full dynamics of the spacetime will be found, and therefore numerical methods must be employed. Only recently have long-term stable numerical evolutions of black-hole binaries been achieved [9]. The flurry of subsequent activity exploring the merger process has so far exclusively focused on rest-mass dominated scenarios (see [10] for a review).

In this Letter we report the first numerical solutions describing the collision of two equal mass BHs in the regime where the initial energy of the system is dominated by the kinetic energy of the BHs. First we describe the problem setup, including the numerical code and initial conditions. We also review some existing analytical approximations to aspects of the problem, which will be important both to interpret the numerical results and to give some confidence in extrapolations of the results to 
infinite boost. Our primary results focus on the gravitational waves emitted during the collision. Unless stated otherwise, we use geometrical units $G=c=1$.

Numerical setup and analysis tools.-The numerical simulations presented here have been performed with the LEAN code, described in detail in [11], where head-on collisions of different classes of initial data were compared. Here we exclusively study evolutions of puncture initial data [12] describing two equal mass, nonspinning, boosted BHs colliding with zero impact parameter in the c.m. frame. The initial coordinate separation between the punctures is set to $r_{0}$, and the boosts are prescribed in the form of nonvanishing Bowen-York [13] parameters $\pm P$ for the initial linear momentum of either BH. The Hamiltonian constraint is solved using Ansorg's spectral solver TWOPUNCTURES [14]. The irreducible masses $M_{\text {irr1,2 }}$ of the BHs are estimated from their apparent horizon areas, calculated using Thornburg's apparent horizon finder AHFINDERDIRECT [15]. This enables us to calculate the BH masses $M_{1,2}$ from Christodoulou's [16] relation $M_{1,2}^{2}=$ $M_{\mathrm{irrl}, 2}^{2}+P^{2}$, from which we define the Lorentz boost parameter $\gamma \equiv M_{1,2} / M_{\text {irr1,2 }}$ (cf. [17]). From a numerical point of view, simulations with large values of $\gamma$ are challenging, partly because the Lorentz contraction decreases the smallest length scale that needs to be resolved. Thus mesh refinement is essential, and here it is provided via the CARPET package [18].

We use the Newman-Penrose scalar $\Psi_{4}$ to measure gravitational radiation. At an extraction radius $r$ from the center of the collision we decompose $\Psi_{4}$ into multipole modes $\psi_{l m}$ of the spherical harmonics of spin weight $-2,{ }_{-2} Y_{l m}$, according to $\Psi_{4}(t, r, \theta, \phi)=\sum_{l=2}^{\infty} \sum_{m=-l-2}^{l} Y_{l m}(\theta, \phi) \times$ $\psi_{l m}(t, r)$. Because of the symmetries of this problem, the only nonvanishing multipoles all have even $l, m=0$, and are purely real, corresponding to a single polarization state $h_{+}$. The energy spectrum and luminosity of the radiation are given by

$$
\begin{gathered}
\frac{d E}{d \omega}=\sum_{l} \frac{1}{16 \pi^{2}} \frac{\left|\hat{\psi}_{l 0}(\omega)\right|^{2}}{\omega^{2}} \equiv \sum_{l} \frac{d E_{l}}{d \omega}, \\
\frac{d E}{d t}=\sum_{l} \lim _{r \rightarrow \infty} \frac{r^{2}}{16 \pi}\left|\int_{-\infty}^{t} \psi_{l 0}(\tilde{t}) d \tilde{t}\right|^{2} \equiv \sum_{l} \frac{d E_{l}}{d t},
\end{gathered}
$$

respectively, where a hat denotes Fourier transform.

Our results are affected by three main sources of uncertainties: finite extraction radius, discretization, and spurious initial radiation. We reduce the error arising from the finite extraction radius by measuring the waveform components at several radii, and fitting them to an expression of the form $\psi_{l m}(r, t)=\psi_{l m}^{(0)}(t)+\psi_{l m}^{(1)}(t) / r$. The waveform "at infinity" $\psi_{l m}^{(0)}(t)$ is the quantity reported throughout this work and used to calculate related quantities, such as the radiated energy. The uncertainty in this extrapolated value is estimated by performing a second fit including also a quadratic term $\psi_{l m}^{(2)} / r^{2}$, and taking the difference be- tween the first- and second-order fits. The resulting uncertainty in the radiated energy is typically $\sim 3 \%-5 \%$.

To estimate discretization errors we evolved the most challenging simulation with $\gamma \approx 3$ with resolutions $h=$ $M / 174, M / 209$, and $M / 244$, where $M=M_{1}+M_{2}$. We observe convergence slightly below second order in the total radiated energy, and use a conservative estimate of $10 \%$ for the resulting error near $\gamma \approx 3$, which drops to a few percent in the nonboosted case (cf. [11]).

Finally, the conformally flat puncture data are known to contain spurious gravitational radiation, which increases strongly with boost $\gamma$ (from a few times $10^{-5}$ for BHs at rest, to about $8 \%$ of the total ADM mass of the system for $\gamma \approx 3$ ). In order to extract physically meaningful information, one has to separate the spurious radiation from the radiation generated by the collision itself. This is done by "waiting" for the spurious radiation to pass the last extraction radius, and then discarding the earlier part of the wave signal. For large boosts, the amount of time between the trailing edge of the spurious radiation and the leading edge of the waves emitted during the collision is roughly $r_{0} /\left(4 \gamma^{2}\right)$. Thus, the initial separation required to cleanly extract the emitted signal increases rapidly with $\gamma$. Because large separations require larger computational domains and longer run times, the spurious radiation effectively limits our ability to study very large $\gamma$. With current resources, we were able to use initial separations of up to $66 M$ for $\gamma>2$, leading to an uncertainty in the total radiated energy which grows rapidly with boost, reaching a value of $5 \%$ for $\gamma \approx 3$. By combining all errors, we estimate the total uncertainty in the radiated energy to be about $15 \%$ for $\gamma \approx 3$, about $10 \%$ near $\gamma=2$ and a few percent for simulations with small velocities.

High-energy collisions are uncharted territory for numerical relativity. It is helpful, therefore, to have alternative methods for guidance and consistency checks. Besides Penrose's bound, we will make extensive use of extrapolations of Smarr's "zero-frequency limit" (ZFL) [19] and of point particle (PP) calculations [20], where one considers a small object of mass $m$ colliding with a massive $\mathrm{BH}$ of mass $M_{\mathrm{BH}}$ to linear order in $m / M_{\mathrm{BH}}$.

Results.-We ran a series of simulations from $\gamma=1$ to $\gamma \approx 3$, with initial separations as discussed in the previous section. In all cases the collision results in a single BH plus gravitational radiation; i.e., there is no sign of any violation of cosmic censorship. The final $\mathrm{BH}$ is born highly distorted. We measure the distortion by taking the ratio $\mathcal{C}$ of the proper equatorial to polar circumferences of the common apparent horizon (CAH). For the range of boosts studied here, the peak value is well fitted by the relation $\mathcal{C}^{\text {peak }} \sim 1.5-0.5 / \gamma$. Thus in the large- $\gamma$ limit $\mathcal{C}^{\text {peak }} \sim 1.5$, in agreement with Penrose's result of $\mathcal{C}=\pi / 2$ for a $\mathrm{CAH}$ consisting of two flat disks. After birth, the BH settles down to a Schwarzschild solution, and the gravitational radiation can be described as a superposition of quasinormal modes (QNMs) of the resulting $\mathrm{BH}$. 


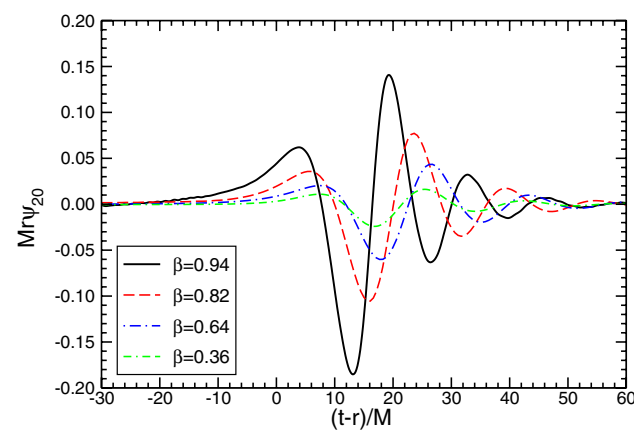

FIG. 1 (color online). Dominant multipolar component $\psi_{20}(t-r)$ for different values of $\beta$, as indicated in the inset.

In Fig. 1 we show the dominant component $\psi_{20}$ of the waveform from collisions with $\gamma=1.07,1.3,1.7,3.0$ (corresponding to $\beta=v / c \simeq 0.36,0.64,0.82,0.94$, respectively). The origin of the $(t-r)$ axis roughly corresponds to the instant of formation of a CAH. One can identify three main parts in the waveforms: a precursor, a main burst at the onset of the CAH formation and the final ringdown tail. These seem to be universal properties of collisions involving BHs and were observed in the past in different settings [20,21]. The start of ringdown, roughly associated with the absolute maxima $\left|\psi_{20}^{\text {peak }}\right|$ in $\left|\psi_{20}\right|$, occurs $\sim 15 M$ after the CAH formation, independently of $\gamma$. Except for a small neighborhood around $\gamma \sim 1$, the maximal wave amplitude $\left|\psi_{20}^{\text {peak }}\right|$ increases monotonically with the boost factor. The small dip in the wave amplitude for small, but nonzero velocities has been seen before both in numerical simulations and analytic predictions [22]. For moderate boosts, we observe the absolute maxima in $\psi_{20}$ to be well approximated by $\left|M r \psi_{20}^{\text {peak }}\right| \approx$ $0.26+0.48 \gamma^{-2}[1 / 4+\log (1 / 2 \gamma)]$ [cf. Eq. (3) below]. The peak amplitude in the waveform $h_{20}$ is roughly $h_{20}^{\text {peak }} \sim$ $\psi_{20}^{\text {peak }} / \omega_{\mathrm{QNM}}^{2}$, where $\omega_{\mathrm{QNM}}$ is the lowest ringdown frequency for the mode [23].

Figure 2 shows the energy spectrum (1) for collisions with different c.m. energy. For large c.m. energies, the spectrum is nearly flat up to some cutoff frequency. A

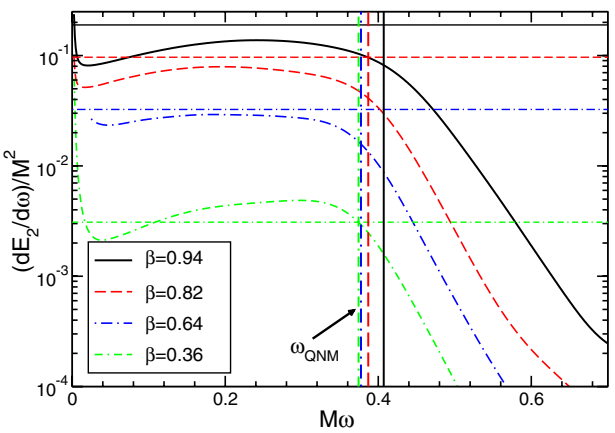

FIG. 2 (color online). Energy spectrum for $l=2$ and different values of $\beta$. Horizontal lines are the corresponding ZFL-PP predictions, vertical lines are the QNM frequencies of the final $\mathrm{BH}$. flat spectrum is predicted by the ZFL and PP approaches, as indicated by the dotted lines in the figure. The cutoff frequency is well approximated by the least-damped QNM of the final hole, marked by a vertical line. The spectrum increases at small frequencies because of initial data contamination and finite-distance effects.

Our numerical results indicate that the peak luminosity (2) is attained approximately $10 \mathrm{M}$ after the CAH formation. The peak luminosity is about $5 \times 10^{-3}$ for $\beta=0.9$, and may be as large as $10^{-2}$ as $\gamma \rightarrow \infty$. Restoring units, we get $10^{-2} c^{5} / G \sim 3.6 \times 10^{57} \mathrm{erg} \mathrm{s}^{-1}$, the largest luminosity from a $\mathrm{BH}$ merger known to date. This is 2 orders of magnitude larger than for the infall from rest of two equal mass BHs, and 1 order of magnitude larger than for the inspiral of equal mass binaries. Nevertheless, it is still 2 orders of magnitude below the universal limit suggested by Dyson, $d E / d t \lesssim 1[24]$.

The total energy $E$ radiated as a function of boost parameter is shown in Fig. 3. Error bars on the radiated energies are determined as described above. We have verified that $E$ calculated from the radiation (2) is consistent with alternative estimates obtained by directly measuring the mass of the final hole from the $\mathrm{CAH}$ properties, and by using the ringdown frequency to estimate the mass of the final hole [23]. The ZFL predicts the following functional form for the total radiated energy as a function of c.m. boost $\gamma$ :

$$
\frac{E}{M}=E_{\infty}\left(\frac{1+2 \gamma^{2}}{2 \gamma^{2}}+\frac{\left(1-4 \gamma^{2}\right) \log \left(\gamma+\sqrt{\gamma^{2}-1}\right)}{2 \gamma^{3} \sqrt{\gamma^{2}-1}}\right)
$$

The quantity $E_{\infty}$ is some unknown cutoff parameter, which is also the total fraction of energy radiated as $\gamma \rightarrow \infty$. By fitting Eq. (3) to the numerical data we obtain $E_{\infty}=$ $0.14 \pm 0.03$. The ZFL is a perturbative calculation about $\omega=0$, and its validity for our scattering problem is not obvious. However, given the good agreement with our numerical results in the kinetic-energy dominated regime $\gamma>2$, the extrapolation procedure should provide a reasonably accurate estimate for $E_{\infty}$.

With regard to the multipolar contributions of the radiated energy, we find that $E_{4}$ is at least 1 order of magnitude smaller than $E_{2}$ for slow-motion collisions. This observa-

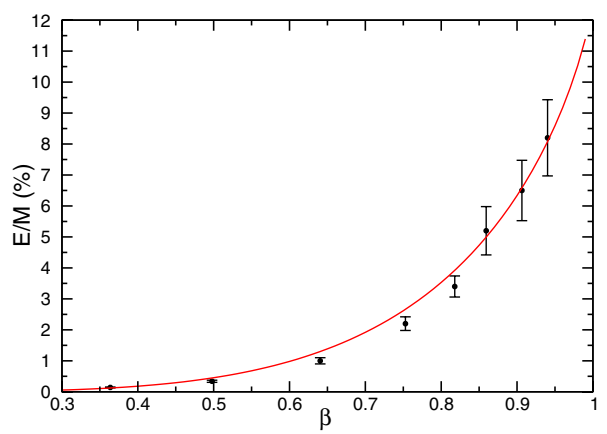

FIG. 3 (color online). Total radiated energy (including error bars) as a function of $\beta$, and best fit using the ZFL prediction. 
TABLE I. Relative multipolar contribution (in \%) and, in parentheses, the ZFL prediction.

\begin{tabular}{ccccccc}
\hline \hline$\beta$ & 0.64 & 0.75 & 0.82 & 0.86 & 0.90 & 0.94 \\
\hline$E_{4} / E_{2}$ & $1.0(1.4)$ & $2.4(3.4)$ & $3.9(5.4)$ & $5.0(7.3)$ & $7.3(10)$ & $11(14)$ \\
$10 E_{6} / E_{2}$ & $0.2(0.3)$ & $1.1(1.5)$ & $2.1(4.0)$ & $4.2(7.5)$ & $11(16)$ & $33(30)$ \\
\hline \hline
\end{tabular}

tion is consistent with the PP results for an infall from rest [20], which predict an exponential decrease of $E_{l}$ with $l$. For larger boosts the ZFL and PP approach predict a strong increase in the relative contribution of higher multipoles, with $E_{l} \sim M / l^{2}$ as $\gamma \rightarrow \infty$. Our numerical results are in reasonable agreement with these calculations, as demonstrated in Table I. The discrepancies still present are due to the relatively large uncertainties in the energy carried by higher multipoles and to the breakdown of the ZFL prediction for small boosts.

Conclusions.-In 1971, Hawking [25] placed an upper limit of $29 \%$ on the total energy radiated when two BHs, initially at rest, coalesce. Numerical simulations of Einstein's equations [21] later showed that the true value is around $0.1 \%-2$ orders of magnitude smaller than Hawking's bound. Using a similar area theorem argument, Penrose [7] derived an upper bound of $29 \%$ for ultrarelativistic head-on collisions (that the numerical values of the two bounds agree is apparently just a coincidence). Here we have presented results indicating that the answer in the high-energy limit is $0.14 \pm 0.03$, slightly less than a factor of 2 of Penrose's bound, though quite close to the estimate of D'Eath and Payne computed using perturbative techniques [26]. Even though our calculations are in $4 \mathrm{D}$, a consequence of this to searches for $\mathrm{BH}$ formation at the LHC is a warning that estimates of the "missing energy" based upon trapped surface calculations could significantly overestimate this effect.

This long overdue study represents an important step towards a full understanding of high-energy $\mathrm{BH}$ collisions. More accurate evolutions using significantly larger boosts are mainly inhibited by the junk radiation in the initial data. More work is also needed to study scattering with a nonzero impact parameter, unequal masses, and nonzero spins. For applications to LHC and RHIC physics, including the effects of extra dimensions, charge and AdS asymptotics (for RHIC) will be necessary.

This work was supported in part by DFG grant $\mathrm{SFB} / \mathrm{TR}$ 7, by FCT-Portugal through projects PTDC/FIS/64175/ 2006 and POCI/FP/81915/2007, and by the Fulbright Foundation (V.C.). E. B. was supported by the NASA Postdoctoral Program at JPL/Caltech, administered by Oak Ridge Associated Universities through a contract with NASA. F. P. was supported by the Alfred P. Sloan Foundation and NSF PHY-0745779. Computations were performed at LRZ Munich, Milipeia at CFC in Coimbra, and the Woodhen cluster at Princeton University.
[1] T. Banks and W. Fischler, arXiv:hep-th/9906038; D. M. Eardley and S. B. Giddings, Phys. Rev. D 66, 044011 (2002); E. Kohlprath and G. Veneziano, J. High Energy Phys. 06 (2002) 057.

[2] N. Arkani-Hamed, S. Dimopoulos, and G. R. Dvali, Phys. Lett. B 429, 263 (1998); I. Antoniadis et al., Phys. Lett. B 436, 257 (1998).

[3] L. Randall and R. Sundrum, Phys. Rev. Lett. 83, 3370 (1999); 83, 4690 (1999).

[4] S. B. Giddings and S. D. Thomas, Phys. Rev. D 65, 056010 (2002); S. Dimopoulos and G. Landsberg, Phys. Rev. Lett. 87, 161602 (2001); J. L. Feng and A. D. Shapere, Phys. Rev. Lett. 88, 021303 (2001).

[5] J. M. Maldacena, Adv. Theor. Math. Phys. 2, 231 (1998); E. Witten, Adv. Theor. Math. Phys. 2, 253 (1998); S. S. Gubser, I. R. Klebanov, and A. M. Polyakov, Phys. Lett. B 428, 105 (1998).

[6] H. Nastase, arXiv:hep-th/0501068; A.J. Amsel, D. Marolf, and A. Virmani, J. High Energy Phys. 04 (2008) 025; S.S. Gubser, S.S. Pufu, and A. Yarom, arXiv:0805.1551 [Phys. Rev. D (to be published)].

[7] R. Penrose, Cambridge University Seminar, Cambridge, England, 1974 (unpublished); D. M. Eardley and S. B. Giddings, Phys. Rev. D 66, 044011 (2002).

[8] P. C. Aichelburg and R. U. Sexl, Gen. Relativ. Gravit. 2, 303 (1971).

[9] F. Pretorius, Phys. Rev. Lett. 95, 121101 (2005); M. Campanelli, C.O. Lousto, P. Marronetti, and Y. Zlochower, Phys. Rev. Lett. 96, 111101 (2006); J. G. Baker, J. Centrella, D. I. Choi, M. Koppitz, and J. van Meter, Phys. Rev. Lett. 96, 111102 (2006).

[10] F. Pretorius, arXiv:0710.1338.

[11] U. Sperhake, Phys. Rev. D 76, 104015 (2007).

[12] S. Brandt and B. Brügmann, Phys. Rev. Lett. 78, 3606 (1997).

[13] J. M. Bowen and J. W. York, Phys. Rev. D 21, 2047 (1980).

[14] M. Ansorg, B. Brügmann, and W. Tichy, Phys. Rev. D 70, 064011 (2004).

[15] J. Thornburg, Phys. Rev. D 54, 4899 (1996); Classical Quantum Gravity 21, 743 (2004).

[16] D. Christodoulou, Phys. Rev. Lett. 25, 1596 (1970).

[17] G. B. Cook and J. W. York, Jr., Phys. Rev. D 41, 1077 (1990).

[18] Carpet Code homepage, http://www.carpetcode.org/.

[19] L. Smarr, Phys. Rev. D 15, 2069 (1977).

[20] M. Davis, R. Ruffini, W. H. Press, and R. H. Price, Phys. Rev. Lett. 27, 1466 (1971); C. O. Lousto and R. H. Price, Phys. Rev. D 55, 2124 (1997); V. Cardoso and J.P. S. Lemos, Phys. Lett. B 538, 1 (2002).

[21] P. Anninos, D. Hobill, E. Seidel, L. Smarr, and W. M. Suen, Phys. Rev. Lett. 71, 2851 (1993).

[22] J. G. Baker et al., Phys. Rev. D 55, 829 (1997).

[23] E. Berti, V. Cardoso, and C. M. Will, Phys. Rev. D 73, 064030 (2006).

[24] F. Dyson, Interstellar Communication, edited by A. G. W. Cameron (Benjamin, NY, 1963), as quoted by K.S. Thorne, in Gravitational Radiation, edited by $\mathrm{N}$. Deruelle and T. Piran (North-Holland, Amsterdam, Netherlands, 1983).

[25] S. W. Hawking, Phys. Rev. Lett. 26, 1344 (1971).

[26] P. D. D'Eath and P. N. Payne, Phys. Rev. D 46, 694 (1992). 